\title{
Other Properties and Presentation of Group from Kronecker Product on the Representation Quaternion Group
}

\author{
Yanita \\ Department of Mathematics, Faculty of Mathematics and Natural Sciences, Andalas University, \\ Kampus Unand Limau Manis Padang 25163, Indonesia
}

\begin{abstract}
The group was obtained by apply Kronecker product on the demonstration of the quaternion group is a finite group with 32 order. The elements of this group are $4 \times 4$ matrices and the group is a non-abelian. In the first results, it's presented that the group was a solvable group. In this paper, other properties will be presented and will also be given a presentation of the group. This property is associated with a series normal subgroup and the cyclic subgroup. It's shown that the presentation of group have 4 generators and 5 relations.
\end{abstract}

Keywords: Series Normal Subgroup, Cyclic Subgroup, Group Presentation.

\section{INTRODUCTION}

We review group $G^{i}$ in Ref. [1]. The group has 32 elements, where elements are $4 \times 4$ matrices. Among the 73 proper subgroups, 19 subgroups with 2 orders, 15 subgroups with 4 orders, 34 subgroups with 8 orders, and 4 subgroups with 16 orders. In addition, there are 25 cyclic subgroups; 19 subgroups with 2 orders and 6 subgroups with 4 orders.

That group has 32 conjugation classes and every element is a conjugation class. ${ }^{a}$ Thus, $K_{A_{i}}=\left\{A_{i}\right\}$ dengan $i=1,2, \ldots, 32$. Elements of $G$ that commute with every element $A_{1}=I_{4}$ (identity matrix with 4 orders) and $A_{2}=-A_{1}$, so we have central of $G$ or $Z(G)=\left\{A_{1}, A_{2}\right\}$.

The paper is organised as follows. In Section 2, we list properties of group $G$. In Ref. [1], it has been explained that group $G$ has solvable and this section other properties are given. These properties use series normal subgroup and cyclic subgroup. In Section 3 we explain about presentation group of $G$.

\section{PROPERTIES OF GROUP $G$}

It has been stated that there are 25 cyclic subgroups of $G$. All factor groups that occur from cyclic subgroups are not cyclic. This proves that the group $G$ is not metacyclic. ${ }^{b}$

\footnotetext{
${ }^{a}$ Let $x$ is element of group $G$, then conjugation class of $x$, denoted by $K_{x}$, is set of elements of $G$ that conjugate with $x$. (Element $x$ and $y$ of $G$ are said conjugate if there is $g$ of $G$ such that $\mathrm{gxg}^{-1}=y$ ) [2].

${ }^{b}$ If there is a normal subgroup cyclic $M$ of group $G$ and factor group $G / M$ is cyclic too, so we called $G$ is metacyclic [3]
}

Since all subgroups of $G$ are normal, there are many series normal subgroups in this group. However, there is no cyclic factor group from these series. This proves that this group is not supersolvable ${ }^{c}$ and not polycyclic. ${ }^{d}$

It's known that $Z(G)=\left\{A_{1}, A_{2}\right\}$, then defined $Z_{1}(G)=Z(G)$ and $Z_{i+1}(G)=\left[Z_{i}(G), G\right]$, where $\left[Z_{i}(G), G\right]=\left\{[A, B] \mid A \in Z_{i}(G), B \in G\right\}$. Consider that $Z_{1}(G)=Z(G)=\left\{A_{1}, A_{2}\right\}, \quad Z_{2}(G)=\left[Z_{1}(G), G\right]=$ $\left\{A_{1}, A_{2}\right\}, Z_{3}(G)=\left[Z_{2}(G), G\right]=\left\{A_{1}, A_{2}\right\}, \ldots, Z_{n}(G)=$ $\left[Z_{n-1}(G), G\right]=\left\{A_{1}, A_{2}\right\}$. It's shown that there are no integers $n$ such that $Z_{n+1}(G)=\left\{A_{1}\right\}$, so this group is not nilpotent. $^{e}$

\section{PRESENTATION OF $G$}

Group presentations are a way of defining groups using generators and relations. These generators and relations are related to the elements in the group. If $P$ be a presentation of group $G$, then each element in $G$ can be presented with one of the generator and relation in the presentation [6]. Generators and relations in this presentation are related

\footnotetext{
${ }^{c}$ Let $H_{i}, i=0,1, \ldots, k$ are subgroup normal in group $G$, if there is a series normal subgroup $\{e\}=H_{0} \subset H_{1} \subset H_{2} \subset \cdots \subset H_{k}=G$, and $H_{i+1} / H_{i}$ is cyclic, then $G$ is supersolvable [2].

${ }^{d}$ Let $H_{i}, i=0,1, \ldots, k$ are subgroup normal in group $G$, if there is a series normal subgroup $\{e\}=H_{0} \subset H_{1} \subset H_{2} \subset \cdots \subset H_{k}=G$ where for each $0 \leq i<k, H_{i}$ is normal in $H_{i+1}$, and $H_{i+1} / H_{i}$ is cyclic for each $i>0$, then $G$ is polycyclic [5].

${ }^{e} \mathrm{~A}$ group $G$ is nilpotent if there is a integer $n$ such that $Z_{n+1}(G)=\{e\}[6]$.
} 
to a term called "word." Therefore, first, the definition of word is given.

Let $X=\left\{x_{1}, x_{2}, \ldots, x_{n}\right\}$ be a set of distinct elements and $X^{-1}=\left\{x_{1}{ }^{-1}, x_{2}{ }^{-1}, \ldots, x_{n}{ }^{-1}\right\}$ be a set of elements, distinct from each other and from the element of $X$. Define $X^{ \pm 1}=X \cup X^{-1}$. A word $W$ is finite string with form $x_{1}^{\varepsilon_{1}} x_{2}^{\varepsilon_{2}} \ldots x_{n-1}^{\varepsilon_{n-1}} x_{n}^{\varepsilon_{n}}, n \geq 0, x_{i} \in X$ and $\varepsilon_{i}= \pm 1, i=$ $1,2, \ldots, n$. We can also be called a word is string of alphabet from $X^{ \pm 1}$. A word $W$ that are $n$-tuples are supposed to have length $n$, symbolized by $|W|=n$. If the word has length 0 , or $|W|=0$, will be symbolized by 1 and the word is called empty word.
Let $W=x_{1}^{\varepsilon_{1}} x_{2}^{\varepsilon_{2}} \ldots x_{n-1}^{\varepsilon_{n-1}} x_{n}^{\varepsilon_{n}}$. Invers of $W$, denoted by $W^{-1}$, and defined $W^{-1}=x_{n}^{-\varepsilon_{n}} x_{n-1}^{-\varepsilon_{n-1}} \ldots x_{2}^{-\varepsilon_{2}} x_{1}^{-\varepsilon_{1}}$. If $W_{1}=$ $x_{i 1}^{\varepsilon_{1}} \ldots x_{i m}^{\varepsilon_{m}}$ and $W_{2}=x_{j 1}^{\varepsilon_{1}} \ldots x_{j n}^{\varepsilon_{n}}$ are word in $X^{ \pm 1}$, then $W_{1} W_{2}=x_{i 1}^{\varepsilon_{1}} \ldots x_{i m}^{\varepsilon_{m}} x_{j 1}^{\varepsilon_{1}} \ldots x_{j n}^{\varepsilon_{n}}$. It's clear that $|W|=\left|W^{-1}\right|$ and $\left|W_{1} W_{2}\right|=\left|W_{1}\right|+\left|W_{2}\right|$.

Example 3.1. Let $X=\{a, b, c\}$. We have $X^{-1}=$ $\{1 / a, 1 / b, 1 / c\}$ and $X^{ \pm 1}=\{a, b, c, 1 / a, 1 / b, 1 / c\}$. Some word in $X^{ \pm 1}$ are

$$
\begin{gathered}
W_{1}=c^{-1} a a b c^{-1} c b \\
W_{2}=c^{-1} a a b c \\
W_{3}=c^{-1} a a b b c^{-1}
\end{gathered}
$$

${ }^{\mathrm{i}}$ Group $G=\left\{A_{k}=\left[a_{i j}\right] \mid i, j=1,2,3,4, k=1,2, \ldots, 32\right\}$ where

$$
\begin{aligned}
& A_{1}=\left[\begin{array}{llll}
0 & 1 & 0 & 0 \\
0 & 0 & 1 & 0 \\
0 & 0 & 0 & 1
\end{array}\right], A_{2}=\left[\begin{array}{rrrr}
0 & -1 & 0 & 0 \\
0 & 0 & -1 & 0 \\
0 & 0 & 0 & -1
\end{array}\right], A_{3}=\left[\begin{array}{rrrr}
0 & -i & 0 & 0 \\
0 & 0 & i & 0 \\
0 & 0 & 0 & -i
\end{array}\right], A_{4}=\left[\begin{array}{rrrr}
0 & i & 0 & 0 \\
0 & 0 & -i & 0 \\
0 & 0 & 0 & i
\end{array}\right]: \\
& A_{5}=\left[\begin{array}{rrrr}
0 & 1 & 0 & 0 \\
-1 & 0 & 0 & 0 \\
0 & 0 & 0 & 1 \\
0 & 0 & -1 & 0
\end{array}\right], A_{5}=\left[\begin{array}{rrrr}
0 & -1 & 0 & 0 \\
1 & 0 & 0 & 0 \\
0 & 0 & 0 & -1 \\
0 & 0 & 1 & 0
\end{array}\right], A_{7}=\left[\begin{array}{llll}
0 & i & 0 & 0 \\
i & 0 & 0 & 0 \\
0 & 0 & 0 & i \\
0 & 0 & i & 0
\end{array}\right], A_{0}=\left[\begin{array}{rrrr}
0 & -i & 0 & 0 \\
-i & 0 & 0 & 0 \\
0 & 0 & 0 & -i \\
0 & 0 & -i & 0
\end{array}\right], \\
& A_{9}=\left[\begin{array}{rrrr}
i & 0 & 0 & 0 \\
0 & i & 0 & 0 \\
0 & 0 & -i & 0 \\
0 & 0 & 0 & -i
\end{array}\right], A_{10}=\left[\begin{array}{cccc}
-i & 0 & 0 & 0 \\
0 & -i & 0 & 0 \\
0 & 0 & i & 0 \\
0 & 0 & 0 & i
\end{array}\right], A_{11}=\left[\begin{array}{rrrr}
-1 & 0 & 0 & 0 \\
0 & 1 & 0 & 0 \\
0 & 0 & 1 & 0 \\
0 & 0 & 0 & -1
\end{array}\right], A_{12}=\left[\begin{array}{rrrr}
1 & 0 & 1 & 0 \\
0 & -1 & 0 & 0 \\
0 & 0 & -1 & 0 \\
0 & 0 & 0 & 1
\end{array}\right], \\
& A_{13}=\left[\begin{array}{rrrr}
0 & i & 0 & 0 \\
-1 & 0 & 0 & 0 \\
0 & 0 & 0 & -i \\
0 & 0 & i & 0
\end{array}\right], A_{14}=\left[\begin{array}{rrrr}
0 & -i & 0 & 0 \\
i & 0 & 0 & 0 \\
0 & 0 & 0 & i \\
0 & 0 & -i & 0
\end{array}\right], A_{15}=\left[\begin{array}{rrrr}
0 & -1 & 0 & 0 \\
-1 & 0 & 0 & 0 \\
0 & 0 & 0 & 1 \\
0 & 0 & 1 & 0
\end{array}\right], A_{16}=\left[\begin{array}{rrrr}
0 & 1 & 0 & 0 \\
1 & 0 & 0 & 0 \\
0 & 0 & 0 & -1 \\
0 & 0 & -1 & 0
\end{array}\right], \\
& A_{17}=\left[\begin{array}{rrrr}
0 & 0 & 1 & 0 \\
0 & 0 & 0 & 1 \\
-1 & 0 & 0 & 0 \\
0 & -1 & 0 & 0
\end{array}\right], A_{18}=\left[\begin{array}{rrrr}
0 & 0 & -1 & 0 \\
0 & 0 & 0 & -1 \\
1 & 0 & 0 & 0 \\
0 & 1 & 0 & 0
\end{array}\right], A_{10}=\left[\begin{array}{rrrr}
0 & 0 & i & 0 \\
0 & 0 & 0 & -i \\
-i & 0 & 0 & 0 \\
0 & i & 0 & 0
\end{array}\right], A_{20}=\left[\begin{array}{rrrr}
0 & 0 & -i & 0 \\
i & 0 & 0 & i \\
i & 0 & 0 & 0 \\
0 & -i & 0 & 0
\end{array}\right], \\
& A_{21}=\left[\begin{array}{rrrr}
0 & 0 & 0 & 1 \\
0 & 0 & -1 & 0 \\
0 & -1 & 0 & 0 \\
1 & 0 & 0 & 0
\end{array}\right], A_{22}=\left[\begin{array}{rrrr}
0 & 0 & 0 & -1 \\
0 & 0 & 1 & 0 \\
0 & 1 & 0 & 0 \\
1 & 0 & 0 & 0
\end{array}\right], A_{23}=\left[\begin{array}{rrrr}
0 & 0 & 0 & i \\
0 & 0 & i & 0 \\
0 & -i & 0 & 0 \\
-i & 0 & 0 & 0
\end{array}\right], A_{24}=\left[\begin{array}{rrrr}
0 & 0 & 0 & -i \\
0 & 0 & -i & 0 \\
0 & i & 0 & 0 \\
i & 0 & 0 & 0
\end{array}\right] \\
& A_{25}=\left[\begin{array}{llll}
0 & 0 & i & 0 \\
0 & 0 & 0 & i \\
i & 0 & 0 & 0 \\
0 & i & 0 & 0
\end{array}\right], A_{26}=\left[\begin{array}{rrrr}
0 & 0 & -i & 0 \\
0 & 0 & 0 & -i \\
-i & 0 & 0 & 0 \\
0 & -i & 0 & 0
\end{array}\right], A_{27}=\left[\begin{array}{rrrr}
0 & 0 & -1 & 0 \\
0 & 0 & 0 & 1 \\
-1 & 0 & 0 & 0 \\
0 & 1 & 0 & 0
\end{array}\right], A_{26}=\left[\begin{array}{rrrr}
0 & 0 & 1 & 0 \\
0 & 0 & 0 & -1 \\
-1 & 0 & 0 & 0 \\
0 & 1 & 0 & 0
\end{array}\right], \\
& A_{20}=\left[\begin{array}{rrrr}
0 & 0 & 0 & i \\
0 & 0 & -i & 0 \\
0 & i & 0 & 0 \\
-i & 0 & 0 & 0
\end{array}\right], A_{30}=\left[\begin{array}{rrrr}
0 & 0 & 0 & -i \\
0 & 0 & i & 0 \\
0 & -i & 0 & 0 \\
-i & 0 & 0 & 0
\end{array}\right], A_{31}=\left[\begin{array}{rrrr}
0 & 0 & 0 & -1 \\
0 & 0 & -1 & 0 \\
0 & -1 & 0 & 0 \\
-1 & 0 & 0 & 0
\end{array}\right], A_{32}=\left[\begin{array}{llll}
0 & 0 & 0 & 1 \\
0 & 0 & 1 & 1 \\
0 & 1 & 0 & 0 \\
1 & 0 & 0 & 0
\end{array}\right]
\end{aligned}
$$


Consider that $\left|W_{1}\right|=8,\left|W_{2}\right|=5$, and $\left|W_{3}\right|=6$. Word of form $x_{i} x_{i}^{-1}$ or $x_{i}{ }^{-1} x_{i}$ for any $i=1,2, \ldots, n$ named inverse pairs. A word is assumed to be reduced if it holds no inverse pair sub-word. $W_{1}$ is not reduced word because it contain $c^{-1} c$ but $W_{2}$ and $W_{3}$ is reduced word.

Next, it's defined new symbol, $\left(X^{ \pm 1}\right)^{*}$, that is the set of words. It's defined function from $\left(X^{ \pm 1}\right)^{*} \times\left(X^{ \pm 1}\right)^{*}$ to $\left(X^{ \pm 1}\right)^{*}$ by writing the words that are to be multiplied. We have to introduce an equivalence relation in $\left(X^{ \pm 1}\right)^{*}$, to obtain a group from $\left(X^{ \pm 1}\right)^{*}$.

Two word are said to be equivalent if one of the words that can be attained from the former by a finite succession of insertion and deletion of expressions $x_{i} x_{i}^{-1}$ or $x_{i}^{-1} x_{i}$ within the word; $x_{i} \in X$. It will be convenient to refer to the pair $x_{i} x_{i}^{-1}$ or $x_{i}^{-1} x_{i}$ together; therefore when $x_{j}=x_{i}{ }^{-1}$ is in $X^{-1}$, defined $x_{j}^{-1}=\left(x_{i}^{-1}\right)^{-1}=x_{i}$. This is confirmed in the following definition:

Definition 3.1 [7]. Two words $V$ and $W$ are equivalent if one of the word can be achieved from the other by finite a finite succession of insertion and deletion of expressions of the form $x_{i}^{\varepsilon_{i}} x_{i}^{-\varepsilon_{i}}, x_{i} \in X, \varepsilon_{i}= \pm 1$. If $V$ and $W$ equivalent, then symbolized by $V \sim W$.

Clear that relation " " in Definition 3.1 is equivalence relation in $\left(X^{ \pm 1}\right)^{*}$. Let $[W]$ is equivalence class of word $W$, then set of equvalence classes $\left\{[W] ; W \in\left(X^{ \pm 1}\right)^{*}\right\}$ with binary operation $[V][W]=[V W]$ for every $V, W \in\left(X^{ \pm 1}\right)^{*}$ thereby becomes a group. This group is called free group on $X$, and symbolyzed by $F(X)$. The identity element of
$F(X)$ is empty word and inverse of $[W]$ is $\left[W^{-1}\right]$, written $[W]^{-1}=\left[W^{-1}\right]$, for every $W \in\left(X^{ \pm 1}\right)^{*}$. We have the next theorem.

THEOREM 3.1 [7]. Let $X$ be a set and $\left(X^{ \pm 1}\right)^{*}$ is set of words built from $X^{ \pm 1}=X \cup X^{-1}$, then product operation definition $\left(X^{ \pm 1}\right)^{*}$ descent in a well-defined fashion to the set $F(X)$ of equivalence classes of member of $\left(X^{ \pm 1}\right)^{*}$, and $F(X)$ thereby becomes a group.

Suppose that $G$ is a group. If on $G$ is defined a set $X$ as in Theorem 3.1 and there is one or some words in $\left(X^{ \pm 1}\right)^{*}$ which is the same as an empty word called a relator set and symbolized by $R$ ( $R$ is a set of words on $\left(X^{ \pm 1}\right)^{*}$ which is the same as an empty word), then the system $\langle X \mid R\rangle$ is referred to a presentation of $G$, and usually written by $P=\langle X \mid R\rangle$, where $X$ is set of generators and $R$ is set of relations.

In this paper the process of determining the generator and relator for the group $G$ is based on the characteristics of the elements in G. Noted that based on the Cayley table $i i$ of $G$, it's found that there are several types of elements in $G$, that is:

The element that $a^{4}=e: A_{3}, A_{4}, A_{5}, A_{6}, A_{7}, A_{8}, A_{9}$, $A_{10}, A_{17}, A_{18}, A_{25}, A_{26}$. Based on these types of element, it's concluded that there are some elements with $a^{2}=$ $a^{4}=e$ will represent several elements, so that the element with this type is one of the generators of this presentation. Noted that, if the first assumption is taken that the generator of this presentation is two, then this assumption

${ }^{\text {ii }}$ Cayley table of $G$

\begin{tabular}{|c|c|c|c|c|c|c|c|c|c|c|c|c|c|c|c|c|c|c|c|c|c|c|c|c|c|c|c|c|c|c|c|c|}
\hline$\times$ & A1 & $A 2$ & $A 3$ & A4 & AS & $A 6$ & $\Delta D$ & $\Delta B$ & AQ & A10 & A:11 & A.12 & A13 & A14 & A15 & A16 & A17 & A48 & A19 & $A 20$ & A21 & A222 & $A 23$ & A24 & A.25 & A26 & A27 & A.28 & A129 & A30 & A31 & $A 32$ \\
\hline A1 & A1 & $A_{2}$ & $A_{3}$ & A4 & A.5 & 46 & A7 & $A B$ & AQ & A10 & A11 & A12 & $\mathrm{A13}$ & A14 & A15 & A16 & A17 & Ads & A19 & $A 20$ & A21 & A222 & $A 23$ & A24 & A.25 & A26 & A27 & A28 & $A 29$ & $A 30$ & A31 & A32 \\
\hline A2 & $\Delta 2$ & $\mathrm{AL}_{1}$ & $A 4$ & $A B$ & $\Delta, 5$ & $A 5$ & $A B$ & $A 7$ & A10 & $A \theta$ & A.12 & Adil & A14 & Ad13 & A16 & A.15 & A.1B & Ad7 & $A 20$ & A19 & A.22 & A21 & A.24 & $\mathrm{AD2}^{2}$ & A.25 & A25 & $A 2 B$ & A.27 & $\triangle 130$ & A29 & $\triangle B 32$ & ABI \\
\hline$A B$ & $A B$ & $A 4$ & $A 2$ & A1 & A7 & $A B$ & $A 65$ & $A 5$ & A11 & A112 & AID & 40 & A15 & A16 & A14 & Ad13 & A.19 & A20 & A18 & A17 & A.23 & A24 & A222 & A21 & A.27 & A2B & A26 & A.25 & A31 & A332 & $A 30$ & A29 \\
\hline A4 & $\triangle 4$ & $\mathrm{A3}$ & A1 & $A 2$ & $A B$ & $A 7$ & A5 & 46 & A.12 & A11 & $A 9$ & Adlo & A16 & A15 & $\mathrm{A13}$ & A14 & A230 & Ad9 & A17 & A18 & A23 & A23 & A21 & A22 & A28 & A27 & A25 & A26 & $\mathrm{AB2}$ & A31 & A29 & $A 30$ \\
\hline 25 & .95 & $A E$ & $A B$ & $A 7$ & $\overline{A_{2}}$ & $\mathrm{Ad}$ & $A 3$ & 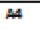 & A.13 & \begin{tabular}{l|l} 
A14 \\
\end{tabular} & A.1 & $\sqrt{15}$ & \begin{tabular}{l|l} 
A10 \\
\end{tabular} & $A 9$ & A11 & Ad2 & A21 & A222 & A24 & 123 & $\overline{Q 226}$ & A.17 & A19 & $A 20$ & 429 & 430 & 132 & A31 & 125 & 225 & 427 & A28 \\
\hline A6 & A6 & 25 & $D 7$ & $A B$ & A1 & 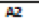 & $\triangle 4$ & $\mathrm{AB}$ & A14 & A13 & A15 & Ad15 & $A 9$ & A10 & A12 & A11 & A.222 & A21 & .123 & A24 & A17 & A18 & $A 20$ & A19 & $A 30$ & A29 & A31 & .432 & 2025 & A226 & A28 & 927 \\
\hline A7 & $\Delta 7$ & $A B$ & A5 & A6 & A44 & $A 3$ & 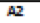 & $\mathbf{A d}$ & 115 & A16 & $\mathrm{A} A \mathrm{AB}$ & Ad4 & A12 & A11 & A10 & $A Q$ & A23 & A24 & A21 & A222 & $\Delta 20$ & A19 & A18 & A17 & AB1 & A32 & A29 & $\mathbf{A 3 0}$ & A28 & A.27 & 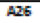 & $A 23$ \\
\hline$A B$ & $A B$ & ${ }^{47}$ & $\Delta 6$ & ${ }^{45}$ & $A B$ & $\$ 4$ & $\Delta 1$ & $A_{22}$ & A16 & A15 & A14 & Al13 & A11 & A12 & $A 9$ & A10 & A23 & A23 & A.22 & A21 & A19 & $A 20$ & A17 & A18 & A32 & AB1 & $\triangle B O$ & A29 & B.27 & A28 & 2025 & A285 \\
\hline A9 & $A 9$ & A10 & A11 & A12 & A:13 & A14 & A15 & A16 & $A 2$ & A1 & DA4 & $\mathrm{AB}$ & A6 & A.5 & $A B$ & $A 7$ & A.23 & A26 & A.27 & A2B & A.29 & A30 & A31 & A32 & A18 & A17 & A20 & A19 & A222 & A21 & A24 & A22 \\
\hline A10 & A10 & $A 9$ & A12 & A11 & A:14 & Ad3 & A16 & Aa15 & A1 & $A 2$ & $A 3$ & A4 & A5 & $A B$ & A7 & $A B$ & A.236 & A25 & A28 & A27 & A330 & A29 & A32 & A31 & A17 & A18 & A19 & A20 & A21 & A.22 & A223 & A24 \\
\hline A11 & A11 & Ad2 & A10 & A9 & A.15 & A16 & A14 & A13 & A,4 & $A B$ & A1 & $\mathrm{AD}^{2}$ & $A B$ & A7 & A5 & $A 6$ & $\mathrm{~A} 227$ & A28 & A26 & $A 25$ & A31 & A32 & $A 30$ & $A 29$ & $A 20$ & A19 & A47 & A18 & A24 & $A 23$ & A21 & A22 \\
\hline A12 & A12 & A.11 & 29 & A10 & A116 & A15 & 13 & Ad4 & $\mathrm{A}_{3} \mathrm{~B}$ & A4 & $A 2$ & 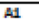 & 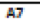 & $A B$ & A6 & A5 & $A 28$ & A27 & $A 25$ & A26 & 32 & A31 & $A 29$ & A30 & 119 & 200 & $\mathrm{~A} 1 \mathrm{~B}$ & A17 & A.23 & A23 & $\$ 222$ & 21 \\
\hline A13 & A13 & A14 & A16 & A15 & A.10 & $A B$ & A11 & A12 & $A 6$ & $A S$ & A7 & $\overline{A B}$ & $\overrightarrow{A 1}$ & $A 2$ & A4 & $A 3$ & A28 & $A B D$ & $\mathrm{ABZ}_{2}$ & A31 & D.266 & A25 & $A 27$ & A28 & A22 & A21 & $\mathrm{A}_{23}$ & A24 & Ad7 & A18 & 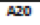 & $\overline{A 19}$ \\
\hline A14 & A14 & A13 & A15 & A15 & $A Q$ & A10 & A12 & Ad1 & AS & $A 6$ & $A B$ & $A 7$ & $A^{2}$ & A1 & $A 3$ & A4 & A30 & A29 & A31 & $A 32$ & 0.25 & A26 & A.28 & A27 & A21 & A22 & $A 2$ & A.23 & Ads & A17 & Ad9 & A200 \\
\hline A15 & A15 & A16 & $\overline{A 13}$ & A14 & A:12 & Ad1 & A10 & $\Delta \theta$ & $A B$ & $A 7$ & $\mathrm{A6}$ & $\Delta S$ & $A B$ & A4 & $\mathrm{A1}$ & 102 & ABH & $A B 2$ & A29 & $A B D$ & A228 & A27 & A236 & A25 & A24 & A23 & A22 & A21 & Ad9 & A20 & Ad7 & A18 \\
\hline A16 & A16 & A15 & A14 & $\mathbf{A 1 3}^{13}$ & Ad11 & Ad12 & $A 9$ & Ad10 & A7 & $A B$ & A.5 & AS & A4 & $A 3$ & $A 2$ & A11 & A332 & AB1 & $A 30$ & A29 & A.27 & A28 & A.25 & A26 & $\mathrm{A}_{23}$ & A24 & A21 & A.22 & A120 & A19 & Ads & A17 \\
\hline A17 & A17 & Ad8 & $\overline{\mathrm{A} 19}$ & $A 20$ & ADZ1 & A.222 & A.23 & A24 & A26 & A25 & $A 28$ & A227 & $A 30$ & A.29 & A32 & A31 & $\mathrm{A} 2^{2}$ & 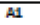 & $\overline{A 4}$ & ${ }^{A 3}$ & $A 6$ & ${ }_{A 5}$ & $A B$ & A7 & A9 & A10 & Ad1 & $\overline{A 12}$ & Ad3 & A14 & Ad15 & 415 \\
\hline A18 & A18 & A17 & A2O & A19 & $A, 22$ & A21 & $A 24$ & A.23 & A.25 & A.21 & $A 27$ & A28 & $A 29$ & A30 & A31 & A32 & $\mathrm{A}_{1}$ & A & 13 & $\Delta$ & 25 & $A B$ & A7 & AB & A10 & $\mathrm{ma}$ & A.12 & 111 & A14 & A:13 & A16 & 415 \\
\hline A19 & A19 & A220 & A18 & A17 & $A 23$ & A24 & $A 22$ & A21 & $A 2$ & $\sqrt{D_{2}}$ & $A 25$ & A256 & $A 32$ & A31 & $\mathrm{Az}^{2}$ & 30 & $\mathrm{AA}^{\mathrm{A}}$ & $\mathrm{AB}_{3}$ & A1 & $A 2$ & $A 8$ & A7 & AS & A6 & A11 & A.12 & A.10 & A9 & 45 & 16 & 14 & 13 \\
\hline A20 & A20 & A49 & A17 & A18 & A.24 & A23 & A21 & A222 & A.27 & A28 & A.26 & A.25 & A31 & A.32 & A30 & A29 & $\mathrm{AB}$ & 44 & $A 2$ & A1 & A7 & $\Delta B$ & A6 & A5 & A12 & A11 & $A 9$ & A10 & A46 & A.15 & Ad3 & A14 \\
\hline A21 & A21 & A.22 & A24 & $A 23$ & A.18 & A.17 & A19 & A20 & $A 30$ & A29 & A31 & A32 & A23 & A.26 & 828 & A227 & A, & A5 & $\Delta 7$ & $A B$ & A1 & $A 2$ & $\mathrm{AA}^{4}$ & $A 3$ & A13 & A14 & A16 & A15 & Ado & $A 9$ & Ad1 & A12 \\
\hline A22 & A.22 & A21 & A23 & A24 & A:17 & A48 & A20 & Ad9 & $A 29$ & A30 & A.32 & ABS & A25 & A25 & A27 & A28 & A.5 & $\Delta 6$ & $A B$ & $\Delta 7$ & $A 2$ & A41 & $\mathrm{AB}_{3}$ & A4 & A14 & A13 & A45 & A16 & $\Delta \theta$ & A10 & Ad2 & A11 \\
\hline A23 & A23 & A24 & A21 & A22 & A.230 & A49 & Q118 & Ad7 & A32 & A31 & A330 & A29 & A27 & A.28 & A225 & 826 & $A B$ & A7 & $\triangle 16$ & 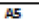 & $\Delta 3$ & A4 & A1 & $A 2$ & A15 & A16 & A413 & A14 & Ad2 & A11 & A10 & A9 \\
\hline A24 & A24 & A23 & A22 & A21 & A.19 & $A 20$ & A17 & Ad8 & ABS & A32 & 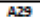 & $A B D$ & $A Z$ & A.27 & A226 & A25 & $A 7$ & $\omega \varepsilon$ & .45 & $A 6$ & ${ }^{A 4}$ & $A 3$ & $\mathrm{~A}_{2}$ & AA & A16 & A15 & A.14 & $\overline{A 13}$ & Ad1 & Ai12 & $\overrightarrow{B Q}$ & 410 \\
\hline A.2S & A25 & A26 & A27 & $A 28$ & A.29 & A30 & AB1 & $A 32$ & A17 & A18 & A.19 & A230 & A21 & A.22 & $A 23$ & A24 & A10 & $\Delta \theta$ & A12 & A11 & A14 & A13 & A16 & A15 & $\mathrm{A}_{2}$ & A1 & $A 4$ & $A 3$ & A6 & $\mathrm{AS}$ & $\mathrm{Ag}$ & $\begin{array}{l}\text { A7 } \\
\end{array}$ \\
\hline A25 & A26 & A25 & A28 & A27 & $A 30$ & A29 & $A 32$ & AB1 & A18 & \begin{tabular}{l|l} 
A17 \\
19
\end{tabular} & $A 20$ & Al19 & $A 22$ & A21 & A23 & A23 & $\mathrm{Ag}$ & AdO & A11 & A12 & $\mathrm{A}_{13}$ & A14 & Ai15 & A15 & A1 & $A_{2}$ & $A B$ & A4 & $A 5$ & $\mathrm{~A} 6$ & ${ }_{A 7}$ & AB \\
\hline A27 & A.27 & A28 & A26 & $A 25$ & A31 & A32 & $A 30$ & A29 & A.19 & A210 & A18 & Ad17 & $A 23$ & A24 & A222 & A21 & A.12 & Ad1 & $A 9$ & A10 & A16 & A.15 & A13 & A14 & A4 & $A B$ & A1 & $A 2$ & $A B$ & A7 & $A 5$ & A6 \\
\hline A28 & A28 & A.27 & A25 & A.26 & A32 & $A 31$ & A29 & A30 & $A 20$ & A19 & A.17 & Ad8 & A24 & A.23 & A21 & A.222 & A11 & Ad2 & A10 & $\triangle 9$ & A.15 & A16 & A14 & A13 & $A 3$ & A4 & $\Delta 2$ & A1 & A7 & $A B$ & A6 & A5 \\
\hline A29 & A29 & A3 & $A 32$ & ABI & A235 & 2025 & A27 & A28 & A21 & A22 & A24 & A273 & A18 & A17 & A19 & A20 & A14 & Ad1 & Q415 & Q116 & $A 9$ & A110 & $A 12$ & A11 & 46 & 4.5 & $\Delta 7$ & $A B$ & $\boldsymbol{B d}$ & $\overline{A 2}$ & $M A$ & A3 \\
\hline A30 & A3O & $\Delta 29$ & $\Delta 31$ & A32 & A.2.5 & $\triangle 126$ & A28 & A.27 & A.22 & A21 & $\Delta, 33$ & N204 & A17 & A18 & $\Delta 20$ & A19 & A:13 & A.44 & A16 & A15 & Q110 & $\mathrm{AB}$ & A11 & A12 & 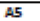 & $\overline{A 6}$ & $A B$ & A7 & 102 & A1 & $\Delta 3$ & A4 \\
\hline A31 & $A 31$ & $A 32$ & A29 & $A 30$ & $\mathrm{~A} 2 \mathrm{23}$ & $\$ 127$ & A26 & A125 & $A 23$ & A23 & A21 & AN22 & $A 20$ & A19 & A1B & A.17 & A.16 & A.15 & A14 & A13 & A11 & A:12 & Ag & A10 & $A B$ & A7 & $A 5$ & A.5 & $\Delta B$ & $A 4$ & $\mathrm{Ad}$ & $\overline{\mathrm{A2}}$ \\
\hline$A 32$ & A32 & $A 31$ & $A 30$ & $A 29$ & A.27 & A.28 & A.25 & A26 & A.24 & A232 & A.22 & A21 & A19 & $A 20$ & A17 & A18 & A.15 & Ad16 & A13 & A14 & A.12 & Ad1 & A10 & $\mathrm{Ag}$ & A7 & $A B$ & A.5 & $A, 5$ & A4 & $A 3$ & $A 2$ & A1 \\
\hline
\end{tabular}


is not right. Furthermore, from Cayley table of $G$, we have $a^{4}$ are $A_{9}$ and $A_{10}$ and $b^{2}$ are $A_{11}, A_{12}, A_{13}, A_{14}$, $A_{15}$, and $A_{16}$. There are $C_{2}^{5}=10$ possible pairs that can be made.

Based on the information and pattern in Cayley table, it's concluded that this presentation was built by more than two generators, that is, it has 4 generators, that is $a, b, c$, and $d$. The relations that can represent all elements are $a^{4}=b^{4}=c^{2}=d^{2}, a^{2}=b^{2}, a b=b a^{-1}$, acd $=$ $d c a^{-1}$ and $b c d=d c b^{-1}$. Thus, presentation of group $G$, is

$$
\begin{gathered}
P=\langle a, b, c, d| a^{4}=b^{4}=c^{2}=d^{2}, a^{2}=b^{2}, a b=b a^{-1}, \\
\left.a c d=d c a^{-1}, b c d=d c b^{-1}\right\rangle
\end{gathered}
$$

So, the elements of $G$ are

$$
\begin{aligned}
& e, a, b, c, d, a^{3}, b^{3}, c^{2}, a b, a^{2} b, a^{3} b, a c, a^{2} c, a^{3} c, a d, \\
& a^{2} d, a^{3} d, b c, a^{2} b c, a^{3} b c, b d, a^{2} b d, c d, a^{2} c d, a^{3} c d, \\
& a b c, a b d, a c d, b c d, a b c d
\end{aligned}
$$

We conclude this paper with the next theorem.
THEOREM 3.2. Group presentation of $G$, is

$$
\begin{aligned}
& P=\langle a, b, c, d| a^{4}=b^{4}=c^{2}=d^{2}, a^{2}=b^{2}, a b=b a^{-1}, \\
& \left.a c d=d c a^{-1}, b c d=d c b^{-1}\right\rangle
\end{aligned}
$$

Proof. Based on (1), it's sufficient to prove this theorem that all elements in $G$ can be represented by (1).

\section{References}

1. Yanita, Y., Helmi, M.R. and Zakiya, A.M., 2019. Solvability group from Kronecker product on the representation of quaternion group. Asian Journal of Scientific Research, 12, pp.293-297.

2. Galian, J.A., 2010. Contemporary Abstract Algebra, 7th edn., USA, Brook/Cole Cengange Learning.

3. Kurosh, A.G., 2003. Theory of Group. USA, American Mathematical Society. Vol. 2.

4. Rotman, J.J., 1999. An Introduction to the Theory of Group, 4th edn., New York, Springer-Verlag.

5. Wehrfritz, B.A.F., 2009. Group and Ring Theoritic Properties of Polycyclic Groups. New York, Springer London Dordrecht Heidelberg.

6. Johnson, D.L., 1997. Presentations of Group, 2nd edn., United Kingdom, Cambridge University,

7. Knapp, A.W., 2006. Basic Algebra. New York, USA, Birkhäuser, Boston, Springer Science+Business Media LLC.

Received: 17 April 2019. Accepted: 24 August 2019. 